\title{
Mesure des variations du tonus pariétal de l'abomasum chez le Mouton
}

\author{
C.H. Malbert et Y. Ruckebusch, \\ avec la collaboration technique de G. Costes et J.P. Serthelon
}

Laboratoire de physiologie associé à I'INRA, 23, Chemin des Capelles, 31076 Toulouse Cedex, France

(reçu le 27-6-1988, accepté le 14-12-1988)

Résumé - Les variations du tonus de la paroi de l'abomasum ont été appréciées par un barostat pneumatique associé à une poche étanche de $250 \mathrm{ml}$ laissée à demeure dans la partie fundique de l'organe chez le Mouton. Le tonus pariétal est évalué à partir de la quantité d'air soustraite ou injectée dans la poche afin d'y maintenir une pression constante de $2 \mathrm{~mm}$ de mercure. Des diminutions périodiques du tonus pariétal apparaissent liées, comme pour le fundus gastrique chez le Chien, aux cycles moteurs du duodénum proximal. Des effets opposés ont été observés à la suite de l'administration de substances cholinomimétiques par rapport à des peptides du groupe de la gastrine, suggérant une sensibilité particulière du tonus de la paroi abomasale aux influences nerveuses et hormonales.

\section{barostat - tonus musculaire - motricité gastro-duodénale}

Summary - Measurement of variations in the abomasal smooth muscle tone in sheep. An inflatable bag of $250 \mathrm{ml}$ at the pressure of $2 \mathrm{~mm} \mathrm{Hg}$ inside the abomasal lumen was used to identify the abomasal smooth muscle tone. Reduced tone of the ovine abomasum was assessed, like for the fundic part of the canine stomach, in relation with the cyclic motor activity of the proximal duodenum. Opposite effects were obtained following parasympathomimetic drug injection versus peptides of the gastrin group, suggesting a high responsiveness of the abomasal smooth muscle tone to both neural and hormonal influences.

barostat - smooth muscle tone - antroduodenal activity

\section{Introduction}

Chez les espèces monogastriques comme le Chien, la partie proximale de l'estomac ou fundus est le siège d'une relaxation dite réceptive au cours d'un repas dont la durée est de quelques minutes. Cette adaptation de la capacité du réservoir gastrique au volume des aliments ingérés correspondrait à une réduction du tonus de la paroi du fundus (Cannon et Lieb, 1911). L'augmentation 
du volume de l'estomac du Chien, contemporaine du repas, qui tend à réduire l'élévation de la pression due à l'arrivée rapide et massive des aliments (Lind et al., 1961; Strunz et Grossman, 1978), a été récemment mesurée à l'aide d'un barostat pneumatique par Aspiroz et Malagelada (1984). Le tonus pariétal, défini par la loi de Hooke, correspond au rapport de la variation de pression au sein d'un organe et des modifications concomitantes de la longueur de sa paroi, c'està-dire du volume du viscère (Stubbs, 1977). L'évaluation du tonus est basée sur l'identité des variations du volume du fundus et de celles d'une poche intérieure remplie d'air dont la pression est maintenue constante, de sorte que l'enveloppe de la poche épouse la paroi du fundus (Aspiroz et Malagelada, 1985).

Chez les espèces polygastriques comme le Mouton, l'existence d'une relaxation analogue pour l'estomac sécrétoire ou abomasum est peu probable. Le réticulo-rumen et le feuillet délivrent, en effet, un flux constant de digesta vers l'abomasum, y compris pendant l'ingestion. Celle-ci dure plusieurs heures et ne semble pas modifier le volume du uréservoir» abomasal ou le débit duodénal par une activité accrue de la "pompe antropylorique" (Ruckebusch et Malbert, 1985). En revanche, chez l'Agneau, le relâchement de l'orifice réticulo-omasal et celui de l'abomasum ont été identifiés au cours de la tétée par Reid et Titchen (1988).

Dans le cas de l'évacuation gastrique d'un repas liquide, le tonus de la paroi du fundus exerce un rôle déterminant, en ce sens que toute variation de pression engendrée par une contraction est proportionnelle à la tension et inversement proportionnelle à la diminution du rayon du viscère (Nelsen et Kohatsu, 1971). L'efficacité propulsive de la contraction de la paroi fundique est ainsi d'autant plus grande que son tonus de base est élevé. Cette influence du tonus de la paroi fundique a été démontrée chez le Chien (Kelly, 1980), et il n'est pas exclu qu'il en soit de même chez le Ruminant adulte, dont le contenu abomasal est toujours liquide. A ce jour cependant, aucun phénomène équivalent n'a été identifié chez le Mouton à partir de jauges de contrainte, aussi bien pour le réticulo-rumen que l'abomasum. Le passage régulier et permanent du contenu réticulo-ruminal vers l'abomasum et la faible épaisseur de la paroi de l'abomasum suggèrent que les éventuelles variations de tonus sont de faible puissance. De surcroît, la surface nécessairement restreinte $\left(>1 \mathrm{~cm}^{2}\right)$, qui est explorée par des jauges de contrainte fixées sur la séreuse, fournit au mieux des oscillations lentes de la ligne de base qui sont de faible amplitude par rapport aux fluctuations énormes et rapides correspondant aux contractions phasiques de la paroi (Ruckebusch et Brady, 1982). II en est de même pour la pression endoluminale évaluée à l'extrémité d'un cathéter perfusé ou à partir de l'électromyogramme global, mais focalisé, de la paroi.

Ce travail a pour objet initial la mesure des variations de tonus de la paroi de l'abomasum chez le Mouton à l'aide d'un barostat pneumatique, selon la technique imaginée par Aspiroz et Malagelada (1985). Dans un second temps, sont examinées les fluctuations spontanées ou provoquées du tonus pariétal de l'abomasum, en particulier pour la motricité périodique dont la jonction gastroduodénale est le siège. Une étape préalable a été la mise au point d'un circuit électronique permettant un asservissement plus précis de la pression que celui utilisé chez le Chien. La validation du système a été effectuée chez le Chien pour être sûr que l'augmentation de la sensibilité n'avait pas été réalisée au détriment de la vitesse de réponse du barostat. 


\section{Matériel et Méthodes}

\section{Barostat pneumatique}

La poche placée dans la lumière de l'abomasum est constituée d'une double enveloppe : l'une de polyuréthane $(0,1 \mathrm{~mm})$ sert d'enveloppe interne étanche à l'air et dépourvue d'élasticité; l'autre de silicone (1 mm) (Silastic, ND) sert d'enveloppe protectrice vis-à-vis des particules alimentaires; celle-ci est nécessaire pour des mesures de longue durée. Un tube à double lumière relie l'intérieur de la poche au barostat proprement dit en vue du maintien d'une pression constante $(2 \mathrm{~mm} \mathrm{Hg})$ au sein de la poche d'un volume initial voisin de $250 \mathrm{ml}$ et du contrôle de cette pression par un système de régulation à boucle fermée (Fig. 1). Un microprocesseur se charge d'acquérir les variations de pression et de les interpréter en vue de les traduire sous la forme de quantité d'air à injecter ou à retirer de la poche à partir d'une seringue. Le logiciel mis au point se charge d'éliminer les oscillations respiratoires présentes sur le signal en provenance du capteur de pression. Son principe est analogue à celui mis au point pour l'analyse en temps réel du débit duodénal chez la brebis (Malbert et Ruckebusch, 1988). Le sens de rotation du moteur pas à pas qui commande la course du piston
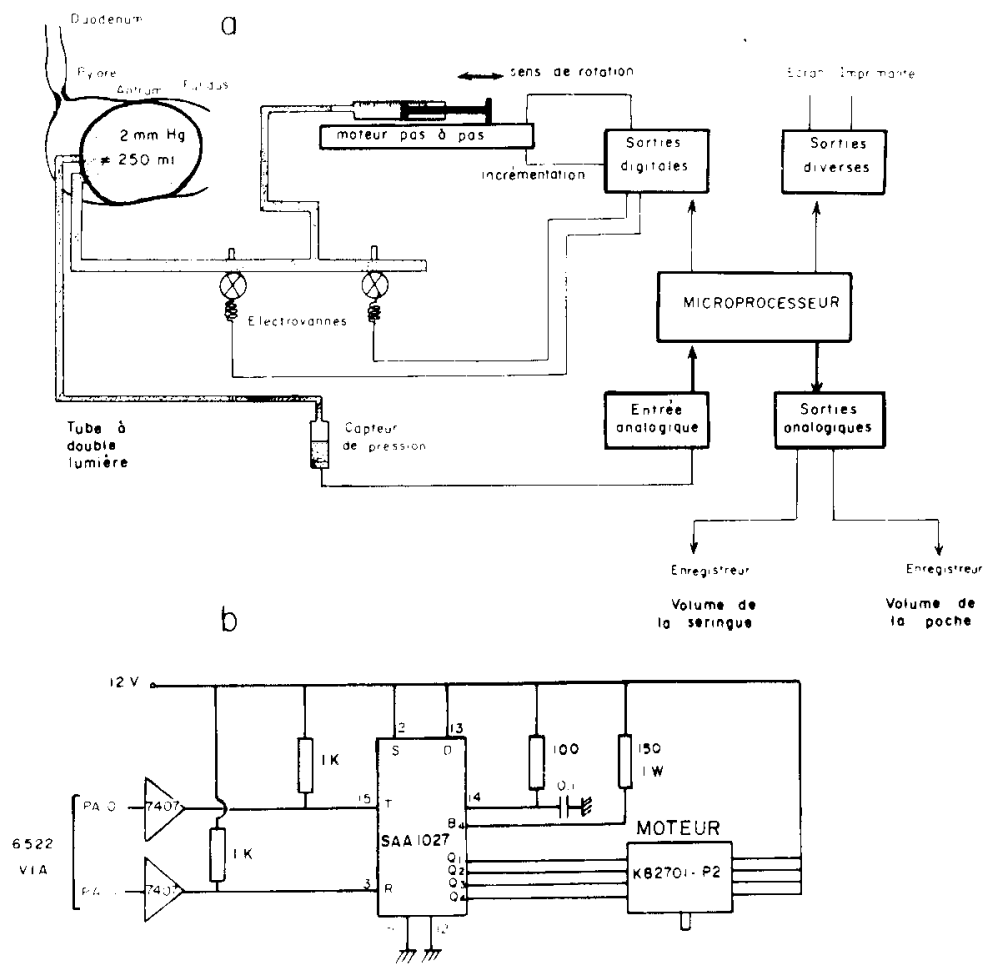

Fig. 1. Principe de mesure en continu des variations du tonus de la paroi de l'abomasum à l'aide d'un barostat pneumatique (a) et électronique de commande du moteur pas à pas, assurant par une seringue le maintien à la pression constante de $2 \mathrm{~mm} \mathrm{Hg}$ l'air contenu dans la poche (b). Le volume de la poche laissée à demeure dans l'abomasum est égal à $250 \mathrm{ml}$ à la pression atmosphérique. L'ensemble de la boucle de rétroaction est régi par un microprocesseur qui interprète les valeurs transmises par le capteur de pression pour les répercuter au moteur pas à pas (circuit SAA 1027) et qui commande l'ouverture ou la fermeture des électrovannes nécessaires à l'équilibre de l'air dans la seringue. L'électronique de commande comporte 2 sorties numériques-analogiques correspondant respectivement au volume de la seringue et à celui de la poche; les 2 convertisseurs D/A correspondants sont connectés aux entrées d'un enregistreur potentiométrique. 
de la seringue s'inverse lorsque la pression devient supérieure à la valeur de consigne haute ou inférieure à la valeur de consigne basse. En cas de surpression, le moteur actionne la seringue de manière à retirer de l'air de la poche. A l'inverse, l'air sera injecté dans la poche si la pression au sein de cette dernière devient insuffisante. Le nombre de pas du moteur est cincrémenté" à chaque point de digitalisation supérieur ou inférieur aux seuils préalablement fixés. La fréquence de digitalisation est fixée par un compteur soustractif relié à la ligne d'interruption du microprocesseur à $10 \mathrm{~Hz}$, soit une valeur plus de dix fois supérieure à celle des contractions de l'abomasum.

L'utilisation d'une seringue de faible volume dans le but d'accroître la résolution du système de mesure s'est révélée inadéquate face à l'importance des variations de volume d'une poche de $250 \mathrm{ml}$ in situ chez le Mouton. Afin de conserver l'avantage d'une forte résolution, nous avons choisi d'augmenter artificiellement le volume de la seringue assurant le remplissage-dégonflage de la poche abomasale par un couple d'électrovannes. En fin de course du piston de la seringue, le microprocesseur commande la fermeture d'une électrovanne "normalement ouverte" placée sur le tube conduisant à la poche, ce qui isole la poche et la maintient sous pression tout au long de l'opération. A ce moment, une électrovanne «normalement fermée" s'ouvre et met le contenu de la seringue en communication avec l'air ambiant pour faire revenir le corps de la seringue en position médiane par une série d'impulsions à fréquence maximale. Cette opération de "recharge" dure 2 sec. Les courbes de l'enregistrement potentiométrique fournissent respectivement les variations à long terme du volume de la poche de 0 a $400 \mathrm{ml}$ et celles à court terme du volume de la seringue.

\section{Mise en place du barostat}

Quatre moutons adultes $(30-35 \mathrm{~kg})$ de race Romanov sont laissés à la diète totale $12 \mathrm{~h}$ avant le début d'une intervention chirurgicale sous anesthésie générale (penthiobarbital sodique - $12 \mathrm{mg} / \mathrm{kg}$ ). L'abomasum est largement extériorisé $(15 \mathrm{~cm})$ sous laparotomie rétrocostale droite. Une incision de la paroi abomasale de $5 \mathrm{~cm}, 10$ à $12 \mathrm{~cm}$ en avant du pylore, permet d'introduire la poche dans la direction de l'orifice omaso-abomasal. Les plans muqueux et musculaires sont refermés à l'aide d'un surjet à points séparés. Le plan séreux est rendu solidaire, à l'aide d'un surjet, d'une rondelle (diamètre $4 \mathrm{~cm}$ ) fixée sur le tube à double lumière en vue d'obtenir une étanchéité parfaite de l'abomasum. L'extrémité libre du tube à double lumière est extériorisée sur le flanc droit en amont de la plaie opératoire. Les poches ont été laissées sur place jusqu'a la perte de leur étanchéité survenue 15 à 35 j après l'opération selon les individus. La position de la poche dans la partie fundique de l'abomasum a été vérifiée à l'autopsie.

Un barostat pneumatique comparable, mais dont le volume était voisin de $400 \mathrm{ml}$, a été utilisé chez le Chien de façon extemporanée. Deux chiens de grand format ( $25 \mathrm{~kg}$ minimum), porteurs d'une fistule chronique du fundus (canule de silicone : diam. int. $4 \mathrm{~cm}$ et diam. ext. $4,5 \mathrm{~cm}$ ) en arrière de l'hypochondre et $10 \mathrm{~cm}$ à droite de la ligne blanche, ont été utilisés. La poche introduite par l'intermédiaire de la canule est laissée à demeure pendant 7 à $8 \mathrm{~h}$.

La sensibilité et la vitesse de régulation de la pression au sein du barostat ont été étalonnées in vitro en plaçant la poche dans une enceinte étanche soumise à des pressions négative ou positive, mimant une diminution ou une augmentation de tonus avant l'implantation chez le Mouton et les enregistrements chez le Chien.

\section{Motricité et transit digestifs}

Deux jauges de contrainte ont été fixées sur la séreuse de l'antre (à 5 ou $2 \mathrm{~cm}$ en amont du pylore) et du fundus $(10 \mathrm{~cm}$ en amont de la jauge antrale) pour mesurer l'activité contractile de la paroi chez les 4 moutons précédents et 2 témoins. Des électrodes souples de $\mathrm{Ni}-\mathrm{Cr}$ ont été fixées dans la paroi duodénale à $20 \mathrm{~cm}$ en aval du pylore de tous les animaux pour identifier les complexes myoélectriques selon des techniques déjà décrites (Malbert et Ruckebusch, 1988). Les jauges de contrainte et leurs coupleurs respectifs sont calibrés avant implantation selon un protocole identique a celui de Gill et al. (1985). La pression intrafundique a été évaluée chez le Chien par la mesure des variations de pression à l'extrémité d'un ballonnet rempli d'eau $(5 \mathrm{ml})$ après introduction à travers la canule.

Chez 2 moutons munis du barostat, une fistule du fundus a été créée en vue du calcul du temps de transit moyen d'un marqueur de la 
phase liquide, le chrome-EDTA, selon la méthode décrite par Gregory et al. (1985). En pratique, une dose de chrome-EDTA ( $2 \mathrm{~g}$ dans $20 \mathrm{ml}$ ) est administrée de 30 en $30 \mathrm{~min}$. Entretemps, la concentration de chrome est évaluée sur des échantillons prélevés de 5 en 5 min par spectrophotométrie d'absorption atomique à $750 \mathrm{~nm}$. Les données obtenues sont comparées à des valeurs témoins (Malbert et Ruckebusch, 1988).

\section{Enregistrements et essais}

Les variations du volume abomasal ont été mesurées chez les moutons recevant 2 repas de foin de graminées par jour ( $9 \mathrm{~h}$ et $17 \mathrm{~h} 30$ ) et disposant d'eau à volonté. Le repas des chiens est constitué d'un mélange homogénéisé de $200 \mathrm{~g}$ d'aliment en boîte et de $200 \mathrm{ml}$ d'eau par jour. La taille moyenne des particules (2 mm) permet l'évacuation en $182 \pm 20 \mathrm{~min}$ de ce repas (Malbert, 1987). L'influence de la prise de nourriture sur les variations de volume gastrique a été également envisagée de la manière suivante : chez le Mouton, présentation de 300 g de granulés au lieu de foin; chez le Chien, double repas correspondant à un volume de $800 \mathrm{ml}$.

Le choix d'une pression interne optimale a été établi en vérifiant les caractéristiques des réponses abomasales à la pression interne du barostat, élevée de 2 à $4 \mathrm{~mm} \mathrm{Hg}$ pendant plusieurs heures chez le Mouton placé dans l'impossibilité de se mettre en décubitus. Les injections intraveineuses de substances gastrokinétiques comme le chlorhydrate de pilocarpine $(5 \mathrm{mg} / \mathrm{kg})$ et le béthanéchol $(10 \mu \mathrm{g} / \mathrm{kg})$, ainsi que celles des hormones peptidiques (pentagastrine : $6 \mu \mathrm{g} / \mathrm{kg}$ et cholécystokinine : 0,12 $\mathrm{pg} / \mathrm{kg}$ ) ont été réalisées tous les jours à la même heure (12 h 30) à la suite d'un enregistrement témoin effectué entre $9 \mathrm{~h}$ et $12 \mathrm{~h} 30$. Dans le cas de la pentagastrine, les mesures ont été également effectuées pour des perfusions à des doses modifiant la motricité antroduodénale.

\section{Résultats}

\section{Caractéristiques du barostat pneuma- tique}

In vitro, une inflation étagée (de 0 à 500 $\mathrm{ml})$ de la poche lorsque la paroi externe est soumise à la pression atmosphérique n'est accompagnée d'aucune variation décelable de la pression au sein de la poche, ce qui confirme sa forte compliance $(C=\Delta V / \Delta P)$. L'inertie du barostat, c'est-à-dire le temps nécessaire à la régularisation de la pression au sein de la poche après une variation de $10 \%$ de la pression de l'enceinte dans laquelle elle est placée, est égale à $0,5 \mathrm{sec}$, valeur à laquelle il convient d'ajouter $0,05 \mathrm{sec}$, c'est-à-dire le temps nécessaire à la mise en marche de la seringue.

In situ, ni l'amplitude, ni la fréquence des contractions fundiques ne sont ralenties par la présence du barostat (Tableau 1). De surcroît, la détection des variations

Tableau I. Caractéristiques contractiles fournies par les jauges de contrainte de la partie fundique et antrale de l'estomac secrétoire du Mouton (1).

$$
\begin{array}{cc}
\text { Fundus } \quad \text { Antrum } & \begin{array}{c}
\text { Statistiques } \\
\text { (ppds) }
\end{array}
\end{array}
$$

$\begin{array}{lrrr}\text { Intervalle entre les contractions (sec) } & 36,7 \pm 24,1 & 83,7 \pm 79,2 & 20,04 \text { * } \\ \text { Durée des contractions (sec) } & 12,3 \pm 4,3 & 4,7 \pm 1,3 & 1,64 \text { * } \\ \text { Amplitude des contractions }(\mathrm{g}) & 1,5 \pm 0,6 & 2,3 \pm 1,1 & 0,45 \text { * }\end{array}$

(1) Moyenne \pm écart type obtenue chez 6 moutons dont 2 témoins pour $6 \mathrm{~h}$ d'enregistrement réparties sur $3 \mathrm{j}$. Nombre de contractions analysées : 2500 .

* Différence significative $(P<0,05)$. 
de pression à partir du barostat se montre plus sensible (Tableau II). Le comportement alimentaire (quantité de matière sèche ingérée et comportement mérycique) n'a pas été perturbé par une poche de $250 \mathrm{ml}$ maintenue à la pression de 2 $\mathrm{mm} \mathrm{Hg}$. Chez l'un des quatre moutons pour lequel l'étanchéité du barostat laissé à demeure a été totale pendant plus d'un mois, la quantité moyenne de foin consommée par jour n'a pas été modifiée. L'augmentation de la pression de 2 à 4 $\mathrm{mm} \mathrm{Hg}$ (de 0,26 à $0,52 \mathrm{kPa}$ ) dans le barostat induit des variations motrices importantes au niveau des jauges de contrainte placées sur le fundus et l'antre (Fig. 2). La pression maximale tolérée sans réponse motrice du fundus est de l'ordre de $2 \mathrm{~mm} \mathrm{Hg}$. Le débit transpylorique des digesta mesuré par la dilution de chrome-EDTA, qui est de $340 \pm 46$ $\mathrm{ml} / \mathrm{h}$ (pour $n=7$ mesures) avec le barostat dégonflé, est de $325 \pm 45 \mathrm{ml} / \mathrm{h}(P>$ $0,05)$ lorsque la pression au sein du barostat est maintenue à $2 \mathrm{~mm} \mathrm{Hg}$.

Variations spontanées du tonus de la paroigastrique

Chez le Chien, les variations de volume du fundus liées à la prise alimentaire sont

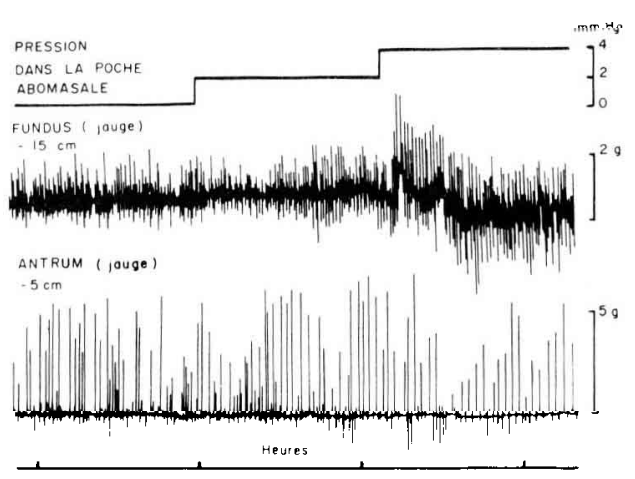

Fig. 2. Réponses motrices du fundus et de l'antre à la distension d'une poche abomasale de $250 \mathrm{ml}$ chez le Mouton. La fréquence des contractions antrales est ralentie alors que les contractions fundiques sont amplifiées par l'addition des $150 \mathrm{ml}$ d'air nécessaires à l'augmentation de la pression à l'intérieur de la poche de 2 à $4 \mathrm{~mm}$ de $\mathrm{Hg}$.

enregistrées de façon plus satisfaisante par le barostat pneumatique que par la pression endoluminale. La durée et l'amplitude de l'augmentation du tonus de la paroi sont fonction de la quantité d'aliments ingérée, la durée moyenne de la relaxation réceptive étant de $12 \pm 5 \mathrm{~min}$ pour un repas de $400 \mathrm{ml}$, et de $24 \pm 9 \mathrm{~min}$ pour un repas de $800 \mathrm{ml}$ pour $n=6$ mesures chez 2 animaux (Fig. 3).

Chez le Mouton, l'activité contractile phasique de la zone fundique consiste en

Tableau II. Motricité fundique enregistrée à partir du capteur de la pression de l'air à l'intérieur de la poche (1).

Jauge de contrainte

(g)

\section{Barostat}

$(\mathrm{mm} \mathrm{Hg})$
Statistiques (ppds)
Intervalle entre les contractions (sec)
$36,7 \pm 34,1$
$15,9 \pm 7,2$
7,88 *
Amplitude des contractions (g ou $\mathrm{mm} \mathrm{Hg}$ ) $\quad 1,5 \pm 0,6$
$2,3 \pm 0,2$
0,2 *

(1) Dans les deux cas, la poche est maintenue à une pression constante de $2 \mathrm{~mm}$ de $\mathrm{Hg}$ par le barostat. Moyenne \pm écart type pour 2 moutons et $4 \mathrm{~h}$ d'enregistrement chacun. Nombre de mesures effectuées : 1500 . "Difference significative $(P<0,05)$. 

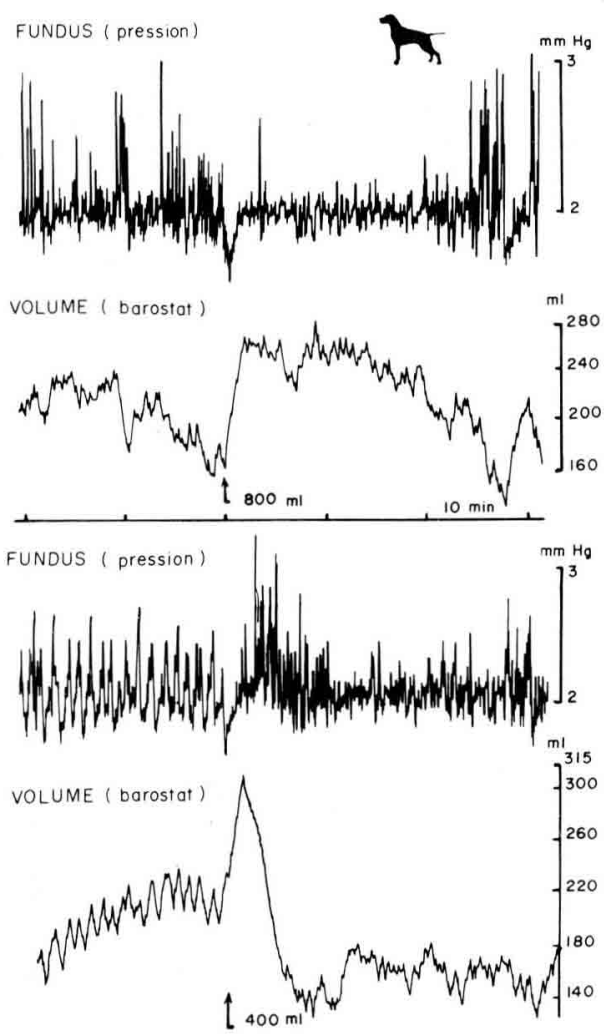

$10 \mathrm{~min}$

Fig. 3. Relaxation réceptive de l'estomac chez le Chien pour un repas semi-liquide de 800 et $400 \mathrm{ml}$. Noter l'amplitude et la durée de la relaxation réceptive en fonction du volume du repas, ainsi que la difficulté d'appréciation du degré de réplétion post-prandiale de l'estomac à partir du tracé des variations de pression.

des contractions régulières plus fréquentes en valeur absolue que celles de l'antre. Le volume du barostat, qui n'est pas modifié par l'ingestion de foin, est réduit de façon transitoire chez les moutons qui consomment avec beaucoup d'avidité $300 \mathrm{~g}$ de granulés. Deux moutons seulement sur 4 ont présenté ce type de réponse à ce repas-friandise (Fig. 4). Enfin, le volume de la poche fundique est accru toutes les 70 à 120 min environ, en
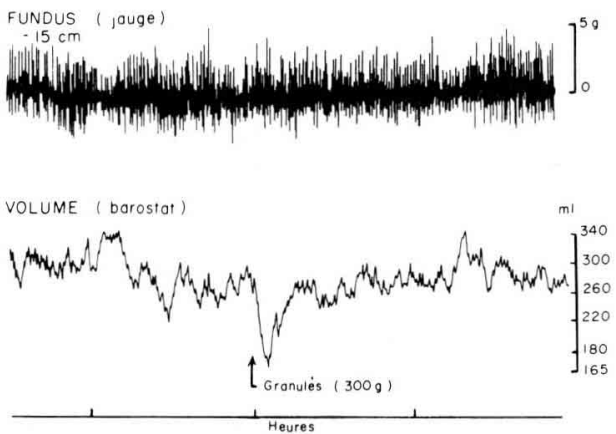

Fig. 4. Augmentation transitoire du tonus pariétal de l'abomasum chez le Mouton à l'ingestion de $300 \mathrm{~g}$ de granulés.

relation avec la période de quiescence faisant suite à l'activité motrice périodique duodénale.

Les variations du volume de la poche fundique sont, les unes, brèves et correspondent aux contractions phasiques; ces contractions, dont la force est supérieure à $1 \mathrm{~g}$, sont immédiatement associées à une diminution du volume de la poche allant de 20 à $35 \mathrm{ml}$; les autres, plus lentes, sont le reflet de variations du tonus de la paroi non associées à des contractions phasiques du fundus. Ces faibles variations du volume de la poche abomasale $(<40 \mathrm{ml}$, soit $16 \%$ du volume de la poche) sont contemporaines d'une période de quiescence sur le duodénum. On observe ensuite une brutale augmentation du volume de la poche de $45-50 \mathrm{ml}$ à la fin de la période d'activité maximale ou activité régulière, qui dure 5-7 min. Au cours de la période de quiescence qui lui fait suite, le volume de la poche demeure constant puis diminue lentement lorsque l'activité duodénale réapparaît au début de la période d'activité irrégulière. Elle atteint alors des valeurs identiques à celles du témoin après 10 à $15 \mathrm{~min}$ (Fig. 5). L'accélération pendant 3 à $4 \mathrm{~h}$ de la fréquence de la motricité périodique du 
FUNDUS ( jauge)

$.5 \mathrm{~cm}$.

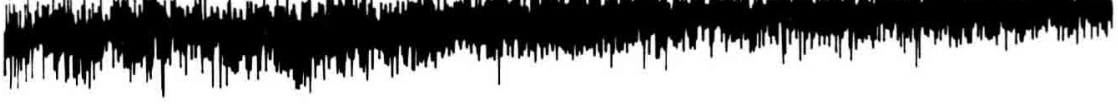
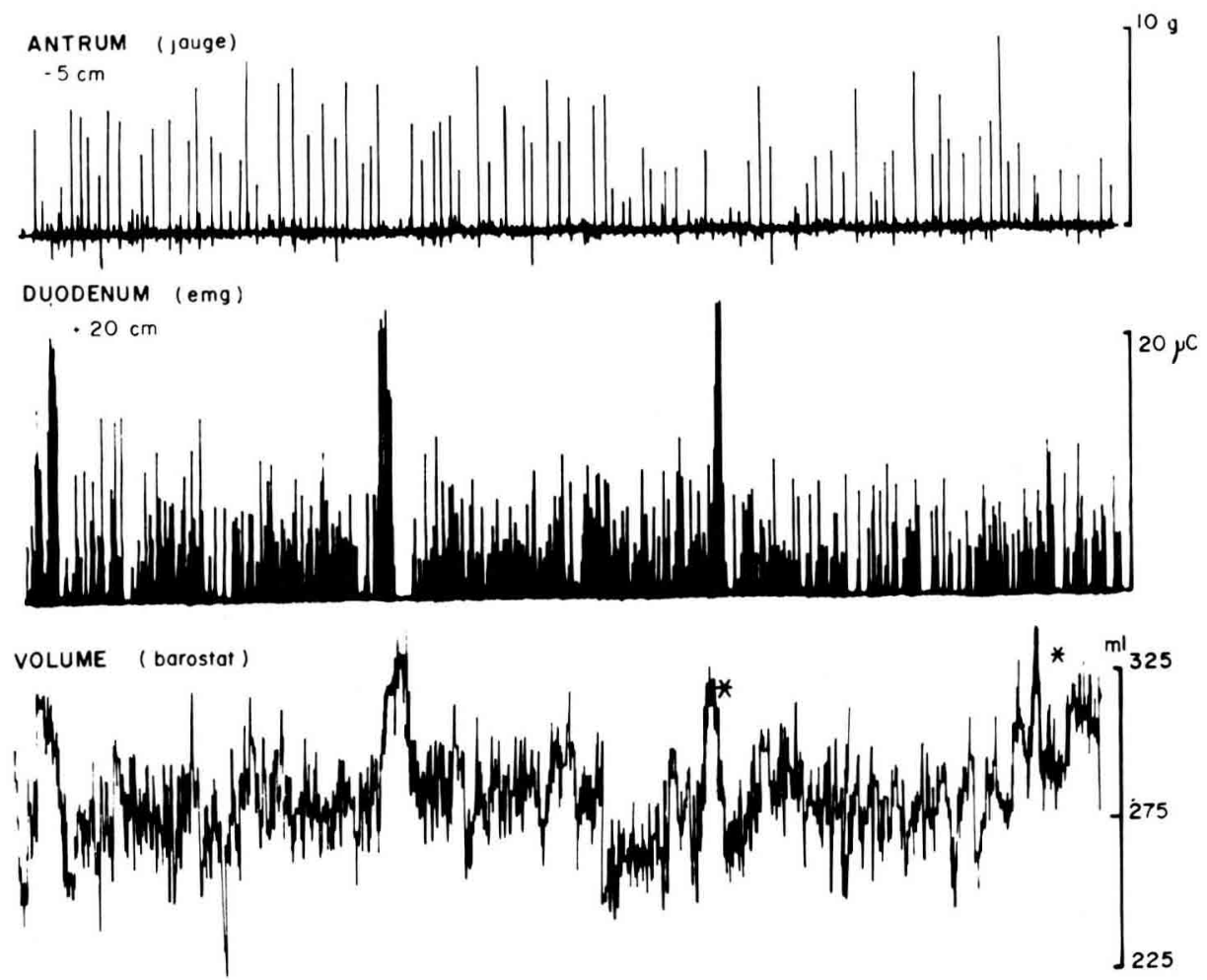

Heures

Fig. 5. Diminution du tonus pariétal de l'abomasum au cours des périodes de quiescence de $3 \mathrm{com}$ plexes myoélectriques migrants présents sur le duodénum proximal. Noter la faible diminution du volume abomasal (") et le retour progressif au volume antérieur dès la reprise de l'activité motrice duodénale.

duodénum, obtenue à la suite de l'administration de méthysergide chez le Mouton (Ruckebusch, 1984), n'a pas été accompagnée de variations concomitantes significatives du tonus pariétal de l'abomasum.
Variations provoquées de la paroi gastrique

L'injection intraveineuse de pilocarpine et celle de béthanéchol sont suivies d'une diminution significative et durable du volume du barostat (Fig. 6). Le volume de la 
poche est réduit de $38 \%$ dans les $5 \mathrm{~min}$ qui suivent l'injection, un effet qui persiste durant $120 \pm 15 \min$ ( $n=6$ mesures). La réduction a été de $25 \%$ pendant $40 \pm 13$ $\min (n=5$ mesures) dans le cas du béthanéchol. La motricité phasique du fundus n'est pas modifiée, contrairement aux contractions antrales qui surviennent de façon ininterrompue à des intervalles de $12-15 \mathrm{sec}$.

Administrée à la forte dose de $1 \mathrm{mg} / \mathrm{kg}$, l'atropine bloque toute motricité phasique, sans altérer de façon significative le tonus pariétal de l'abomasum. Enfin, l'hexamé-

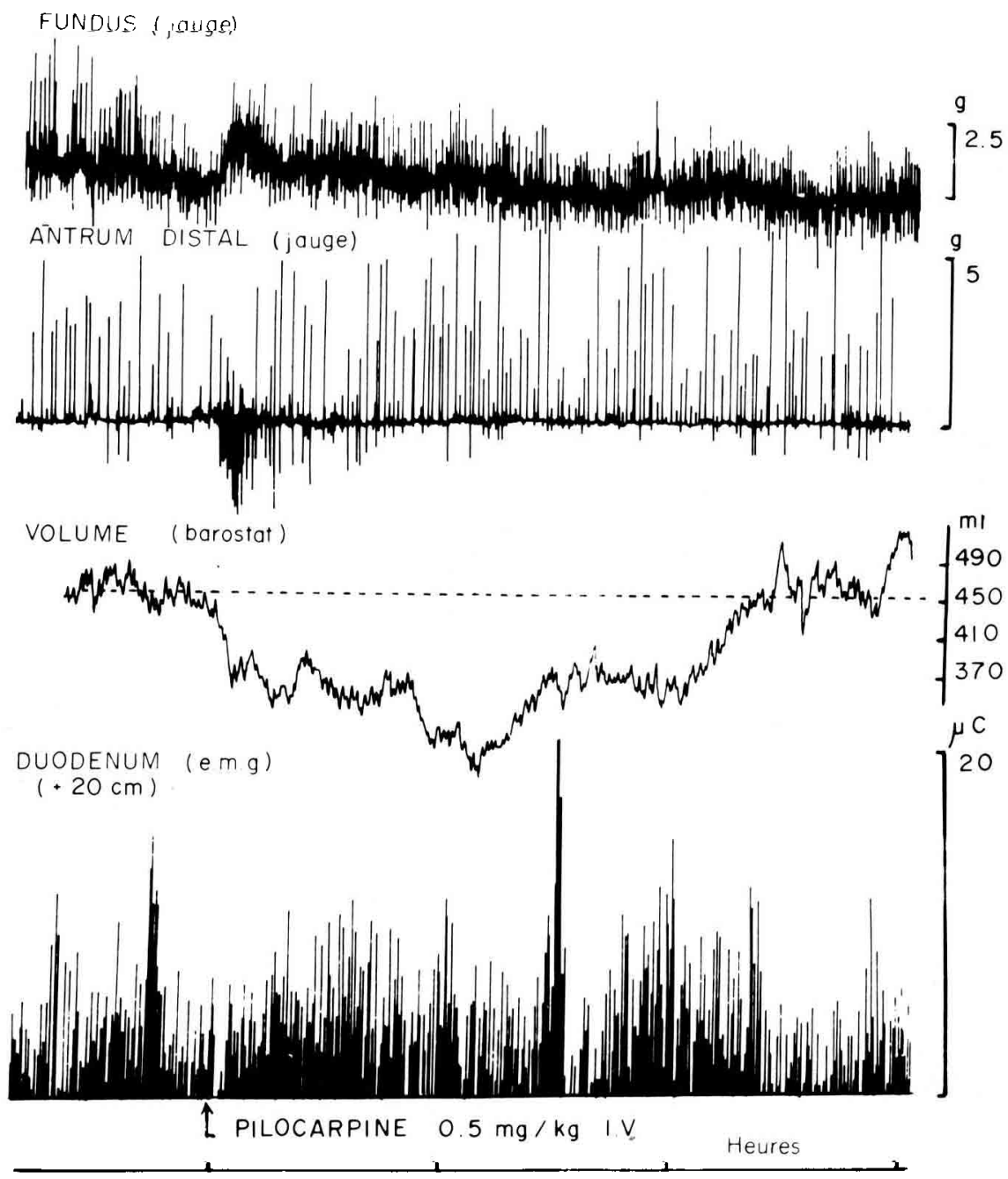

Fig. 6. Effets comparés de la pilocarpine $(0,5 \mathrm{mg} / \mathrm{kg}$, IV) sur la motricité de l'estomac et de l'intestin grêle. Noter la brièveté de l'action de la pilocarpine sur la motricité phasique du fundus, de l'antre et du duodénum comparée à l'augmentation prolongée du tonus pariétal de l'abomasum, caractérisée par la réduction de volume du barostat. 
thonium, un ganglioplégique particulièrement actif chez le Mouton par voie intraveineuse $(2,5 \mathrm{mg} / \mathrm{kg}$ ) (Ruckebusch et al., 1987), augmente le volume de la poche de $33 \%$ environ pendant 2 à $3 \mathrm{~h}$.

Les fragments terminaux de cholécystokinine et de gastrine provoquent une augmentation fugace mais significative du volume de la poche et donc diminuent le tonus basal de l'abomasum. Tout au long de la période au cours de laquelle le tonus est diminué, l'activité phasique du fundus, comme celle de l'antre, sont diminuées (Fig. 7), alors que celle du duodénum proximal est accrue. Un effet similai- re mais accru s'observe au cours de la perfusion intraveineuse de pentagastrine, sans phénomène de rebond à la fin de la perfusion.

\section{Discussion}

\section{Aspects techniques}

Le barostat pneumatique, à l'inverse de ceux mettant en œuvre les déplacements d'eau entre un réservoir à la pression

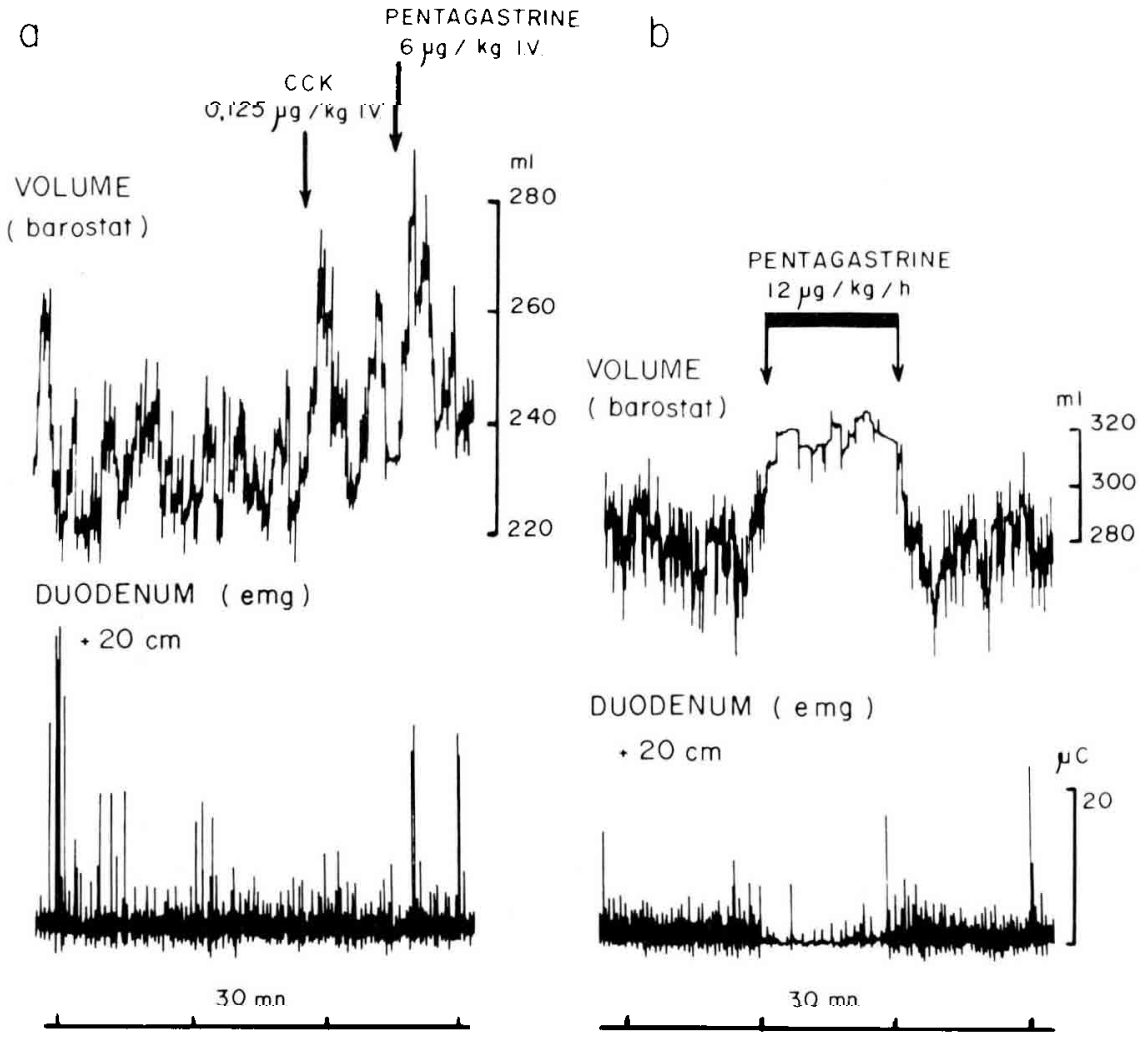

Fig. 7. Diminution du tonus pariétal associée à une légère inhibition de la motricité phasique de l'abomasum à la suite de l'injection de l'octapeptide terminal de la cholécystokinine (CCK) et du pentapeptide actif de la gastrine (a). Un tel effet persiste tout au long d'une perfusion de pentagastrine pendant $30 \mathrm{~min}$ (b). 
atmosphérique et la lumière de l'estomac (Miller et al., 1981), est d'une grande sensibilité. Le travail supplémentaire exigé par la paroi de l'estomac vis-à-vis d'une poche contenant de l'eau est négligeable dans le cas de l'air, comme l'ont montré Chauveau et Marey (1863) pour la mesure des pressions intracardiaques et plus précisément celles de l'oreillette droite chez le Cheval, sur la recommandation du physicien C. Buisson. D'autre part, les faibles résistances à l'écoulement d'un flux d'air expliquent la rapidité des réponses du barostat et sa faible inertie. Par voie de conséquence, la pression intragastrique de référence nécessaire au fonctionnement du barostat peut être faible. L'absence de stimulation ou la stimulation réduite des mécanorécepteurs de l'estomac par le barostat pneumatique pour une pression égale ou inférieure à 2 $\mathrm{mm} \mathrm{Hg}$ est sans doute à l'origine de l'absence de modification du comportement alimentaire chez les brebis avec un barostat laissé à demeure. En effet, selon Grovum (1979), la distension liquide de l'abomasum doit excéder un litre pour réduire l'ingestion alimentaire de $30 \%$ environ chez des moutons de $82 \mathrm{~kg}$. Le volume choisi pour la poche abomasale n'excède pas la moitié du volume de dilution d'un marqueur de la phase liquide dans l'abomasum (Malbert et Ruckebusch, 1988). II apparaît à ce titre comme un compromis puisque la poche gonflée capable de maintenir constante la pression intra-abomasale ne modifie pas le transit antroduodénal. En outre, la localisation de la poche dans la partie fundique de l'abomasum à l'autopsie indique que le tonus enregistré correspond bien à cette partie de l'abomasum et que la fixation de la poche évite toute mobilité excessive sur des périodes de 20 à $30 \mathrm{j}$.

Le coefficient de dilatation thermique plus élevé de l'air comparé à celui de l'eau pourrait être à l'origine d'erreurs de mesure du volume de la poche : l'air y est à la température de $40^{\circ} \mathrm{C}$, alors que celle de l'air injecté est de l'ordre de $20^{\circ} \mathrm{C}$. Pour un écart de température de cet ordre entre la pièce d'une part et celle de l'animal d'autre part, l'augmentation du volume du barostat est de 5,7\%; une telle variation du volume est négligeable vis-àvis des changements d'origine biologique voisins de $30 \%$ au moins.

Le système utilisé pour la mesure du tonus pariétal chez la Brebis reprend la technique utilisée par Aspiroz et Malagelada (1986) pour identifier la relaxation d'origine vagale liée à la présence de nutriments dans le duodénum chez le Chien. Cependant, 2 modifications du système de mesure ont été nécessaires pour une validation interspécifique. Les variations de tonus présentes au niveau de l'abomasum sont très inférieures à celles enregistrées au cours de la prise alimentaire chez le Chien. Une diminution du volume de la seringue par le biais d'électrovannes a donc été nécessaire pour enregistrer des variations faibles et à long terme de ce tonus. L'augmentation de la sensibilité a par ailleurs été réalisée sans diminution de la vitesse de réaction du barostat, qui maintient parfaitement constante la pression intragastrique. Chez le Mouton, la présence d'oscillations respiratoires sur le signal liée à la compression de l'abomasum rend inutilisable le barostat initialement décrit, l'amplitude des oscillations d'origine respiratoire étant équivalente à celle des contractions phasiques du fundus. L'insertion dans le circuit d'asservissement d'un système de suppression en temps réel des variations de pression issues des mouvements respiratoires a permis d'obtenir un tracé significatif des seules variations du tonus.

\section{Aspects physiologiques}

Les variations spontanées de tonus mesurées à l'aide du barostat pneumatique sont remarquables. La diminution du 
tonus du fundus chez le Chien après un repas (et sans doute chez l'Agneau selon Reid et Titchen, 1988) est à l'origine chez cette espèce du temps de latence qui existe entre la fin de la prise de nourriture et le début de l'évacuation gastrique du repas (Hunt et Spurrell, 1951; Stubbs, 1977). Un tel phénomène disparaît après pylorectomie (Malbert et Ruckebusch, 1987) et n'a pas été identifié chez le Mouton puisqu'une réponse de sens inverse existe dans le cas d'un «repas-friandise».

La présence de variations de tonus de la paroi abomasale, concomitantes des phases de quiescence du duodénum, témoigne de l'existence d'une relation nouvelle entre la motricité duodénale et le tonus gastrique. Cette relation concerne non pas la motricité phasique du fundus, qui à l'inverse de celle de l'antre n'est pas altérée par l'apparition des diverses phases du complexe moteur migrant sur l'intestin grêle, mais l'activité tonique. La dissociation entre la motricité phasique de l'antre et l'activité tonique du fundus laisse supposer que le rôle du système nerveux entérique n'est pas primordial. Ce phénomène est du reste étayé par les effets modérés de l'atropine. En revanche, la chute du tonus fundique après une phase d'activité régulière permet de comprendre l'importance de l'arrêt du transit transpylorique du chyme au cours des périodes d'activité régulière et de quiescence développées sur le duodénum (Malbert et Ruckebusch, 1988).

L'analyse des variations provoquées du tonus de l'abomasum à la suite de l'administration d'atropine suggère une faible participation nerveuse cintrinsèque cholinergique" à opposer à l'importance des effets induits à la suite d'une inhibition des synapses ganglionnaires par l'hexaméthonium. Comme le tonus fundique du Chien (Wilbur et Kelly, 1973; Jahnberg et al., 1977; Aspiroz et Malagelada, 1986), le tonus de l'abomasum d'un
Ruminant adulte serait placé sous des influences facilitatrices d'origine vagale susceptibles d'être accrues par l'administration d'agonistes cholinergiques. En effet, le béthanéchol comme la pilocarpine se révèlent capables d'accroître fortement le tonus de l'abomasum. L'existence d'un contrôle d'origine sympathique du tonus abomasal n'est cependant pas exclue, comme le laisse supposer l'importance des effets d'un ganglioplégique tel que l'hexaméthonium. II est possible, mais non démontré, que l'augmentation du tonus perprandial au cours d'un repas-friandise corresponde à une réponse nerveuse centrale.

L'importance des effets des peptides du groupe de la gastrine laisse supposer que ces dernières, libérées au cours de l'acidification du duodénum proximal, c'est-à-dire lorsque le débit transpylorique est maximal, pourraient intervenir indirectement pour réduire le transit antroduodénal. A ce titre, l'un des éléments constitutifs du contrôle du débit pylorique du chyme, serait alors non seulement la pompe "antro-pylorique», mais aussi la pression exercée sur le contenu abomasal par les variations de la tension de la paroi.

\section{Conclusion}

La mesure en temps réel des variations du tonus de l'abomasum chez le Mouton à l'aide d'un barostat pneumatique avec élimination des oscillations d'origine respiratoire indique une analogie avec la partie fundique de l'estomac chez le Chien. L'extrême sensibilité et la faible inertie du barostat pneumatique ainsi que son excellente tolérance locale, sans modifier le transit gastroduodénal, en font un élément fiable et précis de l'appréciation des variations de l'activité tonique de l'aboma- 
sum. Ce type d'activité peut renforcer ou au contraire réduire les effets propulsifs de l'activité phasique.

\section{Références}

Aspiroz F. \& Malagelada J.R. (1984) Pressure activity patterns in the canine proximal stomach : response to distension. Am. J. Physiol. 247, G265-G272

Aspiroz F. \& Malagelada J.R. (1985) Physiological variations in canine gastric tone measured by an electronic Barostat. Am. J. Physiol. 248, G229-G237

Aspiroz F. \& Malagelada J.R. (1986) Vagally mediated gastric relaxation induced by intestinal nutrients in the dog. Am. J. Physiol. 251, G727-G735

Cannon W.B. \& Lieb C.W. (1911) The receptive relaxation of the stomach. Am. J. Physiol. 29, 267-273

Chauveau A. \& Marey P. (1863) Démonstration nouvelle du mécanisme des mouvements du cœur par l'emploi des instruments enregistreurs à indications continues. Mem. Acad. Imp. Med. 26, 269-319

Gill R.C., Pilot P.A., Thomas P.A. \& Wingate W.L. (1985) Organization of fasting and postprandial myoelectrical activity in stomach and duodenum of conscious dogs. Am. J. Physiol. 249, G655-G661

Gregory P.C., Miller S.J. \& Brewer A.C. (1985) The relation between food intake and abomasal emptying and small intestinal transit time in sheep. Br. J. Nutr. 53, 373-380

Grovum W.L. (1979) Factors affecting the voluntary intake of food by sheep. 2 . The role of distension and tactile input from compartments of the stomach. Br. J. Nutr. 42, 425-456

Hunt N.J. \& Spurrell W.R. (1951) The pattern of emptying of the human stomach. J. Physiol. (Lond.) 133, 157-168

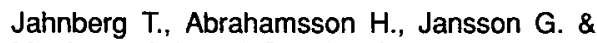
Martinson J. (1977) Gastric relaxatory response to feeding before and after vagotomy. Scand. J. Gastroenterol. 12, 225-228

Kelly K.A. (1980) Gastric emptying of liquids and solids : roles of proximal and distal stomach. Am. J. Physiol. 239, G71-G76
Lind J.F., Duthie H.L., Schlegel J.F. \& Code C.F. (1961) Motility of the gastric fundus. Am. J. Physiol. 201, 197-202

Malbert C.H. (1987) Rôle du pylore dans le transit gastro-intestinal. Thèse Doct. Physiologie animale, Toulouse

Malbert C.H. \& Ruckebusch Y. (1987) Role of pylorus in the control of the canine migrating motor complex. Gastroenterology 92, 19191925

Malbert C.H. \& Ruckebusch Y. (1988) Gastroduodenal motor activity associated with gastric emptying rate in sheep. J. Physiol. (Lond.) 401, 227-239

Miller J., Kauffman G., Elashoff J., Ohashi $H$., Carter D. \& Meyer J.H. (1981) Search for resistances controlling gastric emptying of liquid meals. Am. J. Physiol. 241, G403-G425

Nelsen T.S. \& Kohatsu S. (1971) The stomach as a pump. Rend. R. Gastroenterol. 3, 65-71

Reid A.M. \& Titchen D.A. (1988) Gastric electromyographic activity in the milk-fed lamb. $J$. Physiol. (Lond.) 396, 25-39

Ruckebusch Y. (1984) Enhancement of the cyclic motor activity of the ovine small intestine by lysergic acid derivatives. Mechanism and significance. Gastroenterology 87, 1049-1055

Ruckebusch Y. \& Brady J.C. (1982) Recording and analysis of electrical and mechanical activity of the gastrointestinal tract, $\mathrm{P}$ 209/1-28. In : Techniques in Digestive Physiology (Titchen D.A., ed.). Elsevier Biomedical, County Care, Ireland

Ruckebusch Y. \& Malbert C.H. (1985) Sur la régulation motrice antrodudénale chez le Mouton. Ann. Rech. Vet. 16, 57-68

Ruckebusch Y., Malbert C.H. \& Crichlow E.C. (1987) Hexamethonium : a probe to assess autonomic nervous system involvement in upper gastrointestinal function in concious sheep. Vet. Res. Comm. 11, 293-303

Strunz U.T. \& Grossman M.I. (1978) Effect of intragastric pressure on gastric emptying and secretion. Am. J. Physiol. 235, E552-E555

Stubbs D.F. (1977) Models of gastric emptying. Gut 18, 202-207

Wilbur B.G. \& Kelly K.A. (1973) Effect of proximal gastric, complete gastric and truncal vagotomy on canine gastric electrical activity, motility and emptying. Ann. Surg. 178, 295-303 